\title{
Integralidade em saúde bucal na Atenção Primária à saúde: uma revisão integrativa
}

\author{
Integrality in oral health in primary Health Care: an integrative review \\ Integralidad de la salud bucal en la Atención Primaria de salud: una revisión integradora
}

Recebido: 17/08/2021 | Revisado: 26/08/2021 | Aceito: 30/08/2021 | Publicado: 02/09/2021

\author{
Francisco Jussiê Leal Júnior \\ ORCID: https://orcid.org/0000-0003-4499-9883 \\ Universidade Regional do Cariri, Brasil \\ E-mail: jussieleal@yahoo.com.br \\ Maria Gercileide de Araújo Leal \\ ORCID: https://orcid.org/0000-0002-5392-8940 \\ Universidade Regional do Cariri, Brasil \\ E-mail: gercileide@ hotmail.com \\ José Wagner Martins da Silva \\ ORCID: https://orcid.org/0000-0001-5464-0546 \\ Universidade Estadual do Ceará, Brasil \\ E-mail: wagnermarthins@hotmail.com \\ Águida Raquel Sampaio de Souza \\ ORCID: https://orcid.org/0000-0002-9739-1057 \\ Universidade Regional do Cariri, Brasil \\ E-mail: aguidaraquel.sampaio@gmail.com \\ Maria Corina Amaral Viana \\ ORCID: https://orcid.org/0000-0002-6890-9400 \\ Universidade Regional do Cariri, Brasil \\ E-mail: corina.viana@urca.br \\ Woneska Rodrigues Pinheiro \\ ORCID: https://orcid.org/0000-0003-3353-9240 \\ Universidade Regional do Cariri, Brasil \\ E-mail: woneskar@gmail.com
}

\begin{abstract}
Resumo
O presente estudo teve como objetivo buscar as principais temáticas relativas aos estudos do princípio da integralidade na Atenção Primária à Saúde (APS), numa abordagem relativa à saúde bucal. Trata-se de uma Revisão integrativa da literatura realizada entre os meses de novembro e dezembro de 2020, por meio da Biblioteca Virtual de Saúde, nas bases de dados: Literatura Latino-americana em Ciências da Saúde (LILACS), Medical Literatura Analyses and Retrieval System Online (MEDLINE) e Bibliografia Brasileira de Odontologia (BBO). A amostra final foi de 12 artigos, após o processo de busca e seleção. A atenção em saúde bucal na atenção primária à saúde acontece principalmente através de procedimentos curativos. Os principais procedimentos coletivos são: palestra, atividade de educação em saúde, escovação supervisionada, aplicação tópica de flúor, exame bucal. Os estudos dessa revisão evidenciaram que o acesso às ações de saúde bucal está voltado principalmente às queixas dos pacientes, com procedimentos, na maioria das vezes, curativos e com baixa resolutividade.
\end{abstract}

Palavras-chave: Atenção Primária à saúde; Saúde bucal; Integralidade em saúde.

\begin{abstract}
This study aimed to seek the main themes related to studies on the principle of comprehensiveness in Primary Health Care (PHC), in an approach to oral health. This is an integrative literature review carried out between November and December 2020, through the Virtual Health Library, in the databases: Latin American Literature in Health Sciences (LILACS), Medical Literature Analyzes and Retrieval System Online (MEDLINE) and Brazilian Dentistry Bibliography (BBO). The final sample consisted of 12 articles, after the search and selection process. Oral health care in primary health care takes place mainly through curative procedures. The main collective procedures are: lecture, health education activity, supervised brushing, topical application of fluoride, oral examination. The studies in this review showed that access to oral health actions is mainly aimed at the complaints of patients, with procedures, in most cases, curative and with low resolution.
\end{abstract}

Keywords: Primary Health care; Oral health; Integrality in health.

\section{Resumen}

Este estudio tuvo como objetivo buscar los principales temas relacionados con los estudios sobre el principio de integralidad en la Atención Primaria de Salud (APS), en una aproximación a la salud bucal. Se trata de una revisión integradora de la literatura realizada entre noviembre y diciembre de 2020, a través de la Biblioteca Virtual en Salud, 
en las bases de datos: Literatura Latinoamericana en Ciencias de la Salud (LILACS), Sistema de Análisis y Recuperación de Literatura Médica en Línea (MEDLINE) y Bibliografía de Odontología Brasileña (BBO). ). La muestra final estuvo conformada por 12 artículos, luego del proceso de búsqueda y selección. La atención de la salud bucodental en la atención primaria de salud se realiza principalmente mediante procedimientos curativos. Los principales procedimientos colectivos son: conferencia, actividad de educación sanitaria, cepillado supervisado, aplicación tópica de flúor, examen bucal. Los estudios de esta revisión demostraron que el acceso a las acciones de salud bucal está principalmente dirigido a las quejas de los pacientes, con procedimientos, en la mayoría de los casos, curativos y de baja resolución.

Palabras clave: Atención Primaria de salud; Salud bucal; Integralidad en salud.

\section{Introdução}

A constituição cidadã de 1988 estabelece a implementação do Sistema Único de Saúde (SUS), com base nos princípios doutrinários de universalidade de acesso, integralidade do cuidado e na busca da satisfação equânime das necessidades de saúde da população, apoiados e com sustentação na solidariedade e na compreensão dos determinantes sociais da saúde (Silva; Figueiredo; Gueiros, 2020).

A Política Nacional de Saúde Bucal norteia as ações de saúde odontológica na perspectiva da busca da integralidade da atenção devendo estar voltadas para a amplificação e a capacitação da atenção primária, garantindo o acesso em todos os níveis etários e a oferta dos mais diversos serviços, possibilitando atendimento nos três os níveis de atenção: primário, secundário e terciário (Brasil, 2004).

Para Silva et al (2018a) a integralidade é um princípio e diretriz do SUS que imprime desafios à sua prática, mas que deve ser pautada na procura de atitudes humanizadoras, de respeito e responsabilização de todos os personagens envolvidos no processo do cuidado em saúde. A conduta a ser produzida deve ser fundamentada na integralidade, ocorrendo de forma multidisciplinar e intersetorial, observando as diferenças regionais do Brasil, levando em consideração a cultura, o modo de vida, a religião e o status social dos usuários.

Nesse sentido, a produção desta Revisão Integrativa se dá no intuito de buscar as principais temáticas relativas aos estudos do princípio da integralidade na Atenção Primária à Saúde (APS), numa abordagem relativa à saúde bucal, tendo em vista a melhoria do acesso, a adesão ao tratamento, prevenção, promoção da saúde e reabilitação dos usuários, através de uma compreensão holística do ser humano que em sua completude deve ter um cuidado integral às suas necessidades de saúde odontológicas como parte de um todo indivisível.

O estudo busca identificar produção na literatura acerca da integralidade em saúde bucal, instigando as discussões e aprofundando o conhecimento acerca da temática, visando contribuir para a melhoria da atenção em saúde bucal, através do cuidado integral e da importância de um sistema de saúde que atenda às necessidades do usuário.

\section{Metodologia}

Trata-se de uma revisão integrativa da literatura onde foram seguidas as seguintes etapas fundamentais: elaboração da pergunta norteadora; estabelecimento de critérios para inclusão e exclusão de estudos na literatura; definição das informações a serem extraídas dos estudos; avaliação dos estudos incluídos; interpretação dos resultados e, apresentação da revisão integrativa (Mendes; Silveira; Galvão, 2008).

A questão norteadora desta revisão foi a seguinte: as ações em saúde bucal, na atenção primária, são realizadas de acordo com o princípio da integralidade em saúde?

Buscando facilitar a identificação dos descritores mais adequados para aprimorar e direcionar o processo de busca de respostas apropriadas à pergunta, utilizou-se a estratégia Population, Variables, Outcomes (PVO), onde foram definidas como 
o contexto, atenção primária saúde, como variável de interesse, ações de saúde bucal e o desfecho, a integralidade em saúde, sendo estes os descritores em saúde padronizados para a busca.

As buscas foram realizadas entre os meses de novembro e dezembro de 2020, pareados por dois avaliadores independentes, por meio da Biblioteca Virtual de Saúde (BVS), nas bases de dados: Literatura Latino-americana em Ciências da Saúde (LILACS), MedicalLiteratura AnalysesandRetrieval System Online (MEDLINE) e Bibliografia Brasileira de Odontologia (BBO).

Foram selecionados como critérios de inclusão: estudos primários que abordassem a integralidade da saúde bucal na atenção primária à saúde, em formato de artigo e publicado entre os anos de 2000 e 2020. Justifica-se tal recorte temporal por ter sido no ano de 2000, instituída, pelo Ministério da Saúde, a Portaria 1.444 de 28 de dezembro de 2000, a qual estabelece incentivo financeiro para a reorganização da atenção à saúde bucal prestada nos municípios por meio do Programa de Saúde da Família - PSF (Brasil, 2000).

O critério de exclusão adotado foi: artigos que não responderem à questão norteadora de estudo.

As buscas foram realizadas a partir de quatro cruzamentos dos descritores em saúde: integralidade em saúde, saúde bucal e atenção primária à saúde, com o auxílio do operador booleano AND, na seguinte ordem: "integralidade em saúde AND saúde bucal"; "integralidade em saúde AND atenção primária à saúde"; "atenção primária à saúde AND saúde bucal" e finalmente a busca foi feita com o cruzamento dos três descritores "integralidade em saúde AND saúde bucal AND atenção primária à saúde".

A busca nas bases resultou em 2208 estudos, destes 1339 na LILACS, 664 na MEDLINE e 205 na BBO. Após a aplicação dos filtros, foram elegíveis 1677, conforme estão apresentados em dados quantitativos no Quadro 1.

Quadro 1: Dados quantitativos da busca após aplicação dos filtros nas bases de dados. Crato, CE, 2021.

\begin{tabular}{|l|c|c|c|c|c|}
\hline Bases & $\mathbf{C} * \mathbf{1}$ & $\mathbf{C} * \mathbf{2}$ & $\mathbf{C} * \mathbf{3}$ & $\mathbf{C} * \mathbf{4}$ & Total \\
\hline LILACS & 99 & 593 & 427 & 36 & 1155 \\
\hline MEDLINE & 06 & 26 & 306 & 13 & 351 \\
\hline BBO & 27 & 26 & 105 & 13 & 171 \\
\hline TOTAL & 132 & 645 & 838 & 62 & 1677 \\
\hline
\end{tabular}

$\mathrm{C}^{*}$ refere-se a cruzamento e o número corresponde à ordem de sua realização. Fonte: Autores (2021).

Posteriormente à aplicação de filtros, a etapa seguinte resultou do refinamento em duas fases: 1. a partir da leitura dos títulos e resumos; 2. através da leitura dos artigos na íntegra.

A Figura 1 mostra, de maneira detalhada, o fluxo de seleção dos estudos, com base no instrumento Preferred Reporting Items for Systematic Review and Meta-Analyses (PRISMA). 
Figura 1 - Fluxograma representativo da seleção dos estudos analisados neste estudo. Crato, CE, 2021.

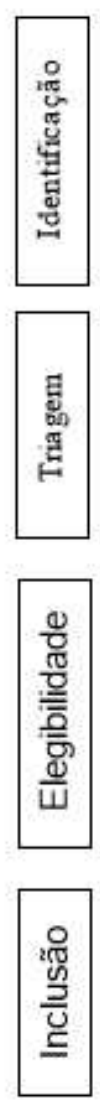

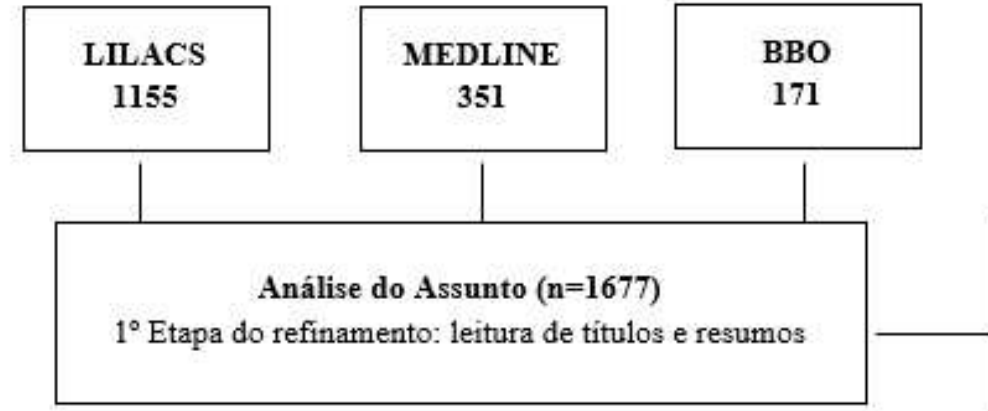

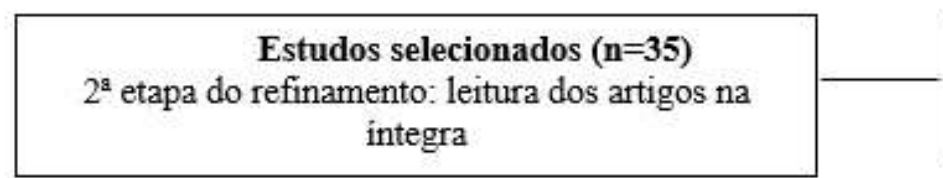

Estudos potencialmente elegíveis ( $\mathrm{n}=16$ )

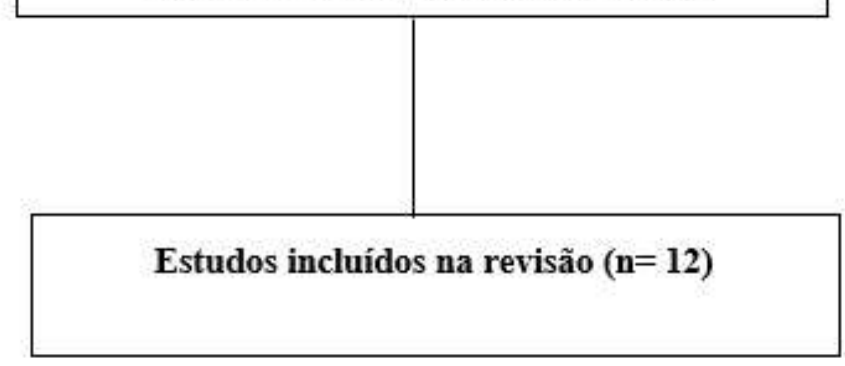

Excluídos:(n=1642)

Não adequação à

temática: 1632

Repetidos: 10

Excluídos: $(n=16)$

Repetidos: 16

Duplicados: 03

Excluídos: (n=04)

Estudos realizados em serviço privado: 04 .

Fonte: Autores (2021).

A etapa de avaliação dos estudos permitiu identificar que de acordo com a classificação da pirâmide de evidências um estudo estava no quinto nível, sendo, estudo quase experimental; oito no sexto, caracterizados como estudos descritivos e três no nível sete de evidências científicas (Sampaio; Mancini, 2007).

\section{Resultados}

Os artigos selecionados, foram apresentados, de forma sintética, no quadro 2, em relação à sua base de dados, autores, ano de publicação, título, desenho do estudo, objetivos e conclusões do estudo. 
Quadro 2 - Características dos estudos quanto à base de dados, os autores, ano de publicação, título, desenho do estudo, objetivos e conclusões. Crato - CE, 2021.

\begin{tabular}{|c|c|c|c|c|c|}
\hline Base/Periódico & $\begin{array}{l}\text { Autores e } \\
\text { ano }\end{array}$ & Título & $\begin{array}{l}\text { Desenho } \\
\text { dos estudos }\end{array}$ & Objetivos & Conclusões dos estudos \\
\hline $\begin{array}{l}\text { LILACS/ } \\
\text { Revista Ciência \& } \\
\text { Saúde Coletiva } \\
\text { (língua } \\
\text { portuguesa) }\end{array}$ & $\begin{array}{l}\text { Neves; } \\
\text { Giordani; } \\
\text { Hugo, } \\
\text { (2019). }\end{array}$ & $\begin{array}{l}\text { Atenção primária à } \\
\text { saúde bucal no Brasil: } \\
\text { processo de trabalho } \\
\text { das Equipes de Saúde } \\
\text { Bucal (ESB). }\end{array}$ & $\begin{array}{l}\text { Transversal, } \\
\text { exploratório. }\end{array}$ & $\begin{array}{l}\text { Avaliar a associação entre } \\
\text { aspectos contextuais } \\
\text { sociodemográficos, indicadores } \\
\text { do sistema de } \\
\text { saúde dos municípios } \\
\text { brasileiros e características do } \\
\text { processo de trabalho e } \\
\text { realização de procedimentos } \\
\text { odontológicos curativos pelas } \\
\text { Equipe de Saúde Bucal } \\
\text { avaliadas durante o primeiro } \\
\text { ciclo do PMAQ-AB no Brasil. }\end{array}$ & $\begin{array}{l}\text { O desempenho da atenção odontológica } \\
\text { curativa no Brasil revelou um cenário de } \\
\text { atenção em saúde bucal distante do } \\
\text { ideal. }\end{array}$ \\
\hline $\begin{array}{l}\text { LILACS/ } \\
\text { Revista Cadernos } \\
\text { de Saúde Púbica } \\
\text { (língua } \\
\text { portuguesa) }\end{array}$ & $\begin{array}{l}\text { Fagundes et } \\
\text { al. (2018). }\end{array}$ & $\begin{array}{l}\text { Diálogos sobre o } \\
\text { processo de trabalho } \\
\text { em } \\
\text { saúde bucal no Brasil: } \\
\text { uma análise com base } \\
\text { no Programa de } \\
\text { Melhoria do Acesso e } \\
\text { da qualidade da } \\
\text { Atenção Básica } \\
\text { (PMAQ-AB). }\end{array}$ & $\begin{array}{l}\text { Estudo } \\
\text { ecológico, de } \\
\text { abrangência } \\
\text { nacional, com } \\
\text { dados } \\
\text { secundários. }\end{array}$ & $\begin{array}{l}\text { Descrever o processo de } \\
\text { trabalho das ESB do Brasil, } \\
\text { com base nos atributos } \\
\text { essenciais da APS, segundo } \\
\text { regiões, tipo de equipe e } \\
\text { características socioeconômicas } \\
\text { dos municípios; verificar se os } \\
\text { dados do processo de trabalho } \\
\text { das ESB do ciclo I do PMAQ- } \\
\text { AB foram capazes de aferir tais } \\
\text { atributos. }\end{array}$ & $\begin{array}{l}\text { As informações sobre o processo de } \\
\text { trabalho das Equipes de Saúde Bucal } \\
\text { foram capazes de discriminar o três } \\
\text { atributos da Atenção Primária à Saúde: } \\
\text { primeiro contato, coordenação do } \\
\text { cuidado e a integralidade na rotina dos } \\
\text { serviços. A longitudinalidade não foi } \\
\text { ratificada. }\end{array}$ \\
\hline $\begin{array}{l}\text { LILACS/ } \\
\text { Revista Gaúcha de } \\
\text { Odontologia } \\
\text { (língua inglesa) }\end{array}$ & $\begin{array}{l}\text { Melo et al. } \\
(2016) \text {. }\end{array}$ & $\begin{array}{l}\text { Avaliação em saúde } \\
\text { bucal na ótica do } \\
\text { gestor municipal. }\end{array}$ & $\begin{array}{l}\text { Estudo } \\
\text { transversal } \\
\text { quantitativo e } \\
\text { qualitativo, } \\
\text { descritivo. }\end{array}$ & $\begin{array}{l}\text { Realizar um diagnóstico } \\
\text { situacional relacionado à } \\
\text { organização dos serviços de } \\
\text { saúde bucal em } 40 \text { municípios } \\
\text { do Estado de São Paulo, Brasil. }\end{array}$ & $\begin{array}{l}\text { Os municípios encontram-se em } \\
\text { distintos momentos na estruturação do } \\
\text { serviço. }\end{array}$ \\
\hline $\begin{array}{l}\text { LILACS / } \\
\text { Physis: Revista de } \\
\text { Saúde Coletiva } \\
\text { (língua } \\
\text { portuguesa) }\end{array}$ & $\begin{array}{l}\text { Moraes; } \\
\text { Klinger- } \\
\text { man; } \\
\text { Cohen, } \\
(2015)\end{array}$ & $\begin{array}{l}\text { Análise do perfil } \\
\text { sociodemo- } \\
\text { gráfico e do processo } \\
\text { de trabalho do } \\
\text { cirurgião-dentista } \\
\text { inserido no PSF em } \\
\text { três municípios da } \\
\text { região serrana do } \\
\text { Estado Rio de Janeiro. }\end{array}$ & $\begin{array}{l}\text { Exploratório, } \\
\text { descritivo. }\end{array}$ & $\begin{array}{l}\text { Analisar o perfil } \\
\text { sociodemográfico } \\
\text { e o processo de trabalho } \\
\text { cirurgião-dentista inserido no } \\
\text { PSF, em três municípios } \\
\text { da região serrana do Estado do } \\
\text { Rio de Janeiro. }\end{array}$ & $\begin{array}{l}\text { Pouco envolvimento dos dentistas com } \\
\text { as condições de vida das famílias e } \\
\text { obstáculos para } \\
\text { trabalhar em equipes multiprofissionais. }\end{array}$ \\
\hline $\begin{array}{l}\text { LILACS/ } \\
\text { Revista Saúde } \\
\text { Debate (língua } \\
\text { portuguesa) }\end{array}$ & $\begin{array}{l}\text { Cassoti et } \\
\text { al. (2014). }\end{array}$ & $\begin{array}{l}\text { Atenção em Saúde } \\
\text { Bucal no Brasil: uma a } \\
\text { análise a partir da } \\
\text { avaliação externa do } \\
\text { Programa de Melhoria } \\
\text { da Qualidade da } \\
\text { Atenção Básica } \\
\text { PMAQ- AB (BA). }\end{array}$ & $\begin{array}{l}\text { Estudo } \\
\text { descritivo e } \\
\text { analítico. }\end{array}$ & $\begin{array}{l}\text { Identificar as características da } \\
\text { oferta de serviços } \\
\text { de odontologia acessados pelos } \\
\text { usuários e analisar a } \\
\text { organização da atenção à saúde } \\
\text { bucal } \\
\text { das equipes participantes do } \\
\text { PMAQ- AB } \\
\text { quanto aos atributos do acesso } \\
\text { de primeiro contato e da } \\
\text { integralidade. }\end{array}$ & $\begin{array}{l}\text { Apenas } 45,1 \% \text { dos usuários conseguem } \\
\text { marcar consulta. A } \\
\text { continuidade de trata } \\
\text { mento é inferior nas regiões Norte e } \\
\text { Nordeste. O acesso e o tempo de espera } \\
\text { nas especialidades revelam problemas } \\
\text { na rede de atenção à saúde bucal. }\end{array}$ \\
\hline $\begin{array}{l}\text { LILACS/ } \\
\text { Revista Brasileira } \\
\text { de Crescimento e } \\
\text { Desenvolvimento } \\
\text { Humano (língua } \\
\text { portuguesa) }\end{array}$ & $\begin{array}{l}\text { Munke- } \\
\text { viz; } \\
\text { Pelicioni, } \\
\text { (2010). }\end{array}$ & $\begin{array}{l}\text { Saúde bucal na } \\
\text { Estratégia Saúde da } \\
\text { Família no } \\
\text { município de São } \\
\text { Paulo: perspectiva do } \\
\text { usuário }\end{array}$ & $\begin{array}{l}\text { Pesquisa } \\
\text { qualitativa. }\end{array}$ & $\begin{array}{l}\text { Identificar as } \\
\text { opiniões, percepções e } \\
\text { necessidades dos usuários } \\
\text { adultos da Unidade Básica de } \\
\text { Saúde Vila } \\
\text { Espanhola, SP em relação à sua } \\
\text { saúde bucal e ao Programa } \\
\text { Saúde da Família. }\end{array}$ & $\begin{array}{l}\text { A inserção do dentista na ESF aumentou } \\
\text { a oferta de serviços e demanda de } \\
\text { procedimentos especializados. As } \\
\text { queixas foram: troca constante de } \\
\text { odontólogos e insatisfação nos critérios } \\
\text { de prioridades no atendimento. }\end{array}$ \\
\hline $\begin{array}{l}\text { LILACS/ } \\
\text { Revista APS - } \\
\text { Atenção Primária } \\
\text { à Saúde (língua }\end{array}$ & $\begin{array}{l}\text { Rodrigues } \\
\text { et al. } \\
\text { (2010), } \\
\text { Feira de } \\
\text { Santana- }\end{array}$ & $\begin{array}{l}\text { Práticas da equipe de } \\
\text { saúde bucal - Equipe } \\
\text { Saúde Bucal na } \\
\text { estratégia saúde da } \\
\text { família e a construção }\end{array}$ & $\begin{array}{l}\text { Estudo } \\
\text { qualitativo. }\end{array}$ & $\begin{array}{l}\text { Analisar as práticas em saúde } \\
\text { bucal na perspectiva da } \\
\text { integralidade no Programa } \\
\text { Saúde da Família de Feira de }\end{array}$ & $\begin{array}{l}\text { Dificuldades no acolhimento e vínculo } \\
\text { devido à rotatividade de profissionais da } \\
\text { Equipe Saúde Família. Resolubilidade } \\
\text { compro }\end{array}$ \\
\hline
\end{tabular}




\begin{tabular}{|c|c|c|c|c|c|}
\hline portuguesa) & BA. & $\begin{array}{l}\text { (des) construção da } \\
\text { integralidade em Feira } \\
\text { de Santana (BA). }\end{array}$ & & Santana- BA. & $\begin{array}{l}\text { metida e problemas na referência e } \\
\text { contrarreferência. }\end{array}$ \\
\hline $\begin{array}{l}\text { LILACS/ } \\
\text { Revista Ciência \& } \\
\text { Saúde Coletiva }\end{array}$ & $\begin{array}{l}\text { Santos; } \\
\text { Assis, } \\
(2006)\end{array}$ & $\begin{array}{l}\text { Da fragmentação à } \\
\text { integralidade: } \\
\text { construindo e } \\
\text { (des)construindo a } \\
\text { prática } \\
\text { de saúde bucal no } \\
\text { Programa de Saúde } \\
\text { da Família de } \\
\text { Alagoinhas, BA. }\end{array}$ & $\begin{array}{l}\text { Qualitativo } \\
\text { perspectiva } \\
\text { histórico- } \\
\text { social. }\end{array}$ & $\begin{array}{l}\text { Analisar os dispositivos que } \\
\text { orientam a atenção integral à } \\
\text { saúde bucal: vínculo, } \\
\text { acolhimento, autonomia, } \\
\text { responsabilização e } \\
\text { resolubilidade. }\end{array}$ & $\begin{array}{l}\text { Atendimento fragmentado, valorização } \\
\text { excessiva da técnica e da especialidade } \\
\text { orientados pelo modelo curativista, } \\
\text { pouca resolubilidade. O acolhimento é } \\
\text { tenso e conflitante, porém com potencial } \\
\text { para transformações positivas. }\end{array}$ \\
\hline $\begin{array}{l}\mathrm{BBO} / \\
\text { Revista Arch } \\
\text { Health Invest } \\
\text { (língua } \\
\text { portuguesa) }\end{array}$ & $\begin{array}{l}\text { Silva et al. } \\
(2018 b)\end{array}$ & $\begin{array}{l}\text { Caracterização do } \\
\text { modelo de atenção } \\
\text { básica à saúde bucal } \\
\text { na região Nordeste no } \\
\text { período de 2015-2017. }\end{array}$ & $\begin{array}{l}\text { Estudo } \\
\text { descritivo e } \\
\text { quantitativo. }\end{array}$ & $\begin{array}{l}\text { Caracterizar o modelo de } \\
\text { atenção básica à } \\
\text { saúde bucal na região Nordeste } \\
\text { do Brasil, a partir de dados } \\
\text { secundários da produção } \\
\text { ambulatorial em } \\
\text { saúde bucal, disponíveis no } \\
\text { SIA-SUS. }\end{array}$ & $\begin{array}{l}\text { O modelo de atenção é } \\
\text { hegemônicamente curativo, com } \\
\text { predomínio de procedimentos restaura } \\
\text { dores, periodontais e cirúrgicos. }\end{array}$ \\
\hline $\begin{array}{l}\text { BBO/ } \\
\text { Revista Ciência } \\
\text { Plural (língua } \\
\text { portuguesa) }\end{array}$ & $\begin{array}{l}\text { Solano et } \\
\text { al. }(2017) \text {. }\end{array}$ & $\begin{array}{l}\text { Utilização dos serviços } \\
\text { de saúde bucal na } \\
\text { Atenção Primária: } \\
\text { perspectivas de } \\
\text { usuários do SUS de } \\
\text { um município do sul } \\
\text { do Brasil. }\end{array}$ & $\begin{array}{l}\text { Estudo } \\
\text { transversal. }\end{array}$ & $\begin{array}{l}\text { Caracterizar e analisar o acesso } \\
\text { ao atendimento odontológico } \\
\text { em Porto Alegre/ RS, por meio } \\
\text { da perspectiva do usuário dos } \\
\text { serviços de saúde da APS do } \\
\text { SUS. }\end{array}$ & $\begin{array}{l}\text { Desconhecimento dos usuários em } \\
\text { relação à existência de serviços } \\
\text { odontológicos curativos e preventivos } \\
\text { na unidade de saúde que acessavam. }\end{array}$ \\
\hline $\begin{array}{l}\mathrm{BBO} / \\
\text { Revista Pesquisa } \\
\text { Brasileira em } \\
\text { Odontopediatria e } \\
\text { Clínica Integrada } \\
\text { (língua } \\
\text { portuguesa) }\end{array}$ & $\begin{array}{l}\text { Cavalcanti } \\
\text { et al. } \\
(2012) \text {. }\end{array}$ & $\begin{array}{l}\text { Avaliação de Usuários } \\
\text { da Atenção Básica } \\
\text { sobre a Implantação de } \\
\text { Protocolo de } \\
\text { Assistência Integral } \\
\text { Odontológica. }\end{array}$ & $\begin{array}{l}\text { Estudo de } \\
\text { intervenção } \\
\text { pesquisa- } \\
\text { ação. }\end{array}$ & $\begin{array}{l}\text { Avaliar a } \\
\text { implantação de um Protocolo } \\
\text { de Assistência Integral } \\
\text { Odontológica (PAIO), segundo } \\
\text { os usuários } \\
\text { assistidos por uma Unidade de } \\
\text { Saúde da Família, na } \\
\text { cidade de João Pessoa- PB - } \\
\text { Brasil. }\end{array}$ & $\begin{array}{l}\text { Os usuários consideram que a } \\
\text { implementação de um PAIO possibilitou } \\
\text { a melhoria do serviço de saúde e da } \\
\text { atenção em saúde bucal, ampliando o } \\
\text { acesso e resolutividade. }\end{array}$ \\
\hline $\begin{array}{l}\text { MEDLINE/ } \\
\text { Revista Plos One } \\
\text { (língua inglesa) }\end{array}$ & $\begin{array}{l}\text { Reis et al. } \\
(2019) \text {. }\end{array}$ & $\begin{array}{l}\text { Compreenden-do o } \\
\text { desempenho da equipe } \\
\text { de saúde bucal na } \\
\text { atenção primária: um } \\
\text { estudo de método } \\
\text { misto. }\end{array}$ & $\begin{array}{l}\text { Estudo de } \\
\text { métodos } \\
\text { mistos com } \\
\text { desenho } \\
\text { explicativo } \\
\text { sequencial. }\end{array}$ & $\begin{array}{l}\text { Descrever as ações de APS } \\
\text { realizadas pelas Equipes de } \\
\text { Saúde Bucal no Brasil e } \\
\text { compreender aspectos que } \\
\text { levam a diferentes níveis de } \\
\text { desempenho em Equipe de } \\
\text { Saúde Bucal. }\end{array}$ & $\begin{array}{l}\text { Dificuldades de acesso; falhas na } \\
\text { prevenção, diagnóstico precoce e } \\
\text { acompanhamento de câncer bucal; } \\
\text { reabilitação protética insuficiente. }\end{array}$ \\
\hline
\end{tabular}

Fonte: Autores (2021).

Muitos estudos realizaram a busca de dados secundários através de informações do Instituto Brasileiro de Geografia e Estatística e Índice de Desempenho do Sistema Único de Saúde (Neves; Giordani; Hugo, 2019), dados do Programa Nacional de Melhoria do Acesso e da Qualidade da Atenção Básica PMAQ - AB (Neves; Giordani; Hugo, 2019; Fagundes, 2018; Cassoti et al., 2014), dados disponíveis no disponíveis no Sistema de Informações Ambulatoriais do Sistema Único de Saúde (Silva et al., 2018b).

Quanto às técnicas utilizadas houve estudos de campo (Moraes; Klingerman; Cohen, 2015); entrevistas (Cassoti et al., 2014; Munkeviz; Pelicioni, 2010; Rodrigues et al., 2010; Santos; Assis, 2006) e análise de conteúdo de Bardin (Munkeviz; Pelicioni, 2010; Moraes; Klingerman; Cohen, 2015).

Os participantes dos estudos foram usuários (Cassoti et al., 2014; Munkeviz; Pelicioni, 2010; Rodrigues et al., 2010), dentistas da atenção básica (Moraes; Klingerman; Cohen, 2015; Cassoti et al., 2014; Rodrigues et al., 2010; Santos; Assis, 2006), coordenadores de saúde bucal (Melo et al., 2016; Santos; Assis,2010), auxiliares de saúde bucal (Rodrigues et al., 2010), outros trabalhadores de saúde (Rodrigues et al., 2010; Santos; Assis, 2010), e informantes-chave: prefeito, secretário de saúde, coordenador da Estratégia de Saúde da Família (Santos; Assis, 2010). 
Os estudos evidenciaram que o serviço prestado é essencialmente curativo (Neves; Giordani; Hugo, 2019; Moraes; Klingerman; Cohen, 2015; Silva et al., 2018b), principalmente no Sudeste e Sul do país (Neves; Giordani; Hugo, 2019).

Entre os procedimentos curativos realizados estão: restaurações em amálgama e resina, exodontias, raspagens, alisamentos, polimentos periodontais, curativos de demora e drenagem de abscessos dentoalveolares (Neves; Giordani; Hugo, 2019), com tratamentos restauradores superando os cirúrgicos (Silva et al., 2018b). Por outro lado, entre as equipes de saúde bucal avaliadas em todo o Brasil, algumas não executam um ou mais dos procedimentos odontológicos de desfecho, quais sejam: restauraç̃̃es em amálgama e resina, exodontias, raspagens, alisamentos e polimentos periodontais, curativos de demora e drenagem de abscessos dentoalveolares (Neves; Giordani; Hugo, 2019).

Os procedimentos e orientações coletivas centradas na higiene bucal são realizados pelos técnicos em higiene bucal, como escovação supervisionada, evidenciação de placa bacteriana e bochecho fluorado (Munkevi Z; Pelicione, 2010). Esses procedimentos são efetivados principalmente em ambiente escolar para crianças de 6 a 14 anos (Melo et al., 2016; Santos; Assis, 2006).

As atividades desenvolvidas pelo profissional odontólogo são, essencialmente, clínicas e dentro do consultório, a maior parte da carga horária (Moraes; Klingerman; Cohen, 2015).

A visita domiciliar é pouco executada, porém, alguns procedimentos como escovação, orientação dos cuidadores, extração, restauração temporária, exame bucal, remoção de sutura, raspagem e colocação de curativo são realizados durante a visita aos idosos, acamados ou pacientes com dificuldade de locomoção (Moraes; Klingerman; Cohen, 2015).

Os atendimentos de saúde bucal são realizados em instituições de convívio coletivo, como escolas (Melo et al., 2016; Santos; Assis, 2006; Moraes; Klingerman; Cohen, 2010), creches e asilos (Moraes; Klingerman; Cohen, 2015). As principais atividades citadas foram: palestra, atividade de educação em saúde, escovação supervisionada, aplicação tópica de flúor, exame bucal com finalidade de estimar as necessidades e separar os grupos de riscos (Moraes; Klingerman; Cohen, 2015).

A abordagem aos usuários dos serviços ocorre através da avaliação de risco e vulnerabilidade no primeiro atendimento sendo reportada como uma atividade do cotidiano da clínica por grande parcela dos participantes, bem como o cuidado continuado, que é garantido através da agenda equipe de Saúde Bucal (Cassoti et al., 2014). Para outros, a demanda é dividida entre os que precisam de atendimento de urgência, normalmente para o alívio da dor, e os que serão agendados, mas não é feito uma classificação de risco, levando os pacientes de algumas equipes aguardarem até um ano pelo atendimento (Reis et al., 2019; Melo et al., 2019; Munkeviz; Pelicioni, 2010; Rodrigues et al., 2010).

A integralidade do processo de trabalho das equipes de saúde bucal perpassa por atendimentos em outros serviços fora da rede de atenção primária e nesse caso, os estudos mostram que quando há necessidade de encaminhamentos para outros serviços, os pacientes são referenciados (Cavalcanti et al., 2012; Fagundes et al., 2018; Reis et al., 2019; Rodrigues et al., 2010), para o Centro de Especialidades Odontológicas (CEO) (Fagundes et al., 2018; Reis et al., 2019).

Em alguns casos é relatada a dificuldade em garantir a continuidade do tratamento, visto que é feita a referência para outros serviços, no entanto não existe uma contrarreferência efetiva e os pacientes não conseguem o atendimento ou não retornam à Atenção Primária à Saúde (Rodrigues et al., 2010); poucas equipes possuem referências para cinco especialidades odontológicas obrigatórias e na maioria das vezes não contam com protocolos para fluxo de tratamento (Fagundes et al., 2018).

A busca de pacientes para tratamento de saúde bucal é realizada por meio de grupos de programas, encaminhamentos pelos Agentes Comunitários de Saúde e utilização de critérios de risco (Melo et al., 2016).

$\mathrm{O}$ acolhimento é reportado de forma diversa: como parte da triagem de usuários, como estratégia de humanização e como uma maneira de envolver todos os integrantes da Atenção Primária da Saúde (Reis et al., 2019), como uma forma 
positiva de resolutividade (Cavalcanti et al., 2012), porém ainda há casos que os profissionais e usuários reconhecem que o acolhimento é limitado ou não existe, nem no seu sentido mais restrito (Rodrigues et al., 2010).

A dificuldade em garantir um cuidado integral no SUS leva o usuário a procurar um serviço privado, seja pra realizar procedimentos de limpeza, profilaxia, raspagem supra gengival, manutenção dos cuidados ou tratamento preventivo, realização de restauração e colocação de prótese, mesmo esses procedimentos estando no escopo da Atenção Primária à Saúde (Solano et al., 2017; Reis et al., 2019) ou para tratamento em serviço de média complexidade (Rodrigues et al., 2010; Reis, et al., 2019).

\section{Discussão}

A integralidade é um dos atributos essenciais da atenção primária à saúde, no entanto, ainda parece ser um dos desafios mais consistentes, e o baixo desempenho deste pode estar relacionado à incapacidade organizacional da Atenção Primária à Saúde para a oferta de serviços e à heterogeneidade dos mesmos (Cunha et al., 2019).

Os achados dessa revisão remetem dificuldades da efetivação da integralidade da saúde bucal (Solano et al., 2017; Reis et al., 2019; Rodrigues et al., 2010; Neves; Giordani; Hugo, 2019; Moraes; Klingerman; Cohen, 2015; Silva et al., 2018b).

Esses dados corroboram com os encontrados por Maciel et al., (2020), que após analisarem os atributos da integralidade na ótica dos usuários, observaram que a avaliação dos serviços odontológicos destinados à comunidade estudada era considerada insatisfatória, pois havia baixa adesão aos serviços de saúde bucal oferecidos, persistindo o modelo de atendimento tradicional evidenciado no pronto atendimento, centrado nos aspectos meramente curativos e mutiladores. Essa prática dificulta a organização em torno da prevenção, promoção e recuperação da saúde preconizada pelo SUS (Barros et al., 2016).

Mesmo quando realizadas, as atividades de prevenção em saúde bucal, trazem, em alguns estudos, um modelo limitado e centrado em uma prática antiga da odontologia, conforme descrito em estudo desenvolvido por Aquilante; Acioli (2013), onde os membros das equipes de saúde bucal ainda centram suas atividades intersetoriais preventivas em palestras e aplicações tópicas de flúor na comunidade ou nas escolas, reproduzindo o modelo incremental de atenção à saúde que foi preponderante nos anos de 1950 a 1980.

Os usuários de um município do interior do Ceará, também qualificaram a atenção da saúde bucal como inadequada aos princípios da Estratégia Saúde da Família, persistindo em um modelo de assistência tradicional, pontual, curativista, biológico e mutilador (Maciel et al., 2020). Tal modelo de atenção contrapõem-se ao que pressupõe um atendimento integral, o qual deve considerar o sujeito como um todo e não apenas determinar o cuidado limitados as suas demandas odontológicas, sejam elas urgentes ou eletivas.

As ações intersetoriais de educação em saúde bucal ainda apresentam baixa cobertura, baseadas em modelos tradicionais como palestras educativas, levantamentos epidemiológicos de necessidades, escovação supervisionada, aplicações tópicas de flúor e bochechos fluoretados, realizados nas Unidades Básicas de Saúde ou em espaços alternativos como escolas, creches, empresas, centros comunitários (Mattos et al., 2014).

É importante destacar atividades e rotinas odontológicas fundamentadas e voltadas uma clínica ampliada em saúde bucal, caracterizada por um acolhimento de escuta ao usuário que produz subjetividades e promove a pactuação de um plano terapêutico, em que a tomada de decisão é compartilhada e está em constante reconstrução, contrário a uma prática essencialmente biológica e centrada na doença do ser humano (Graff; Toass, 2018).

Outro entrave da integralidade da saúde bucal, diz respeito à oferta de consultas odontológicas no SUS. No Brasil, nas equipes de atenção básica, esse serviço ocorre em menos de $80 \%$ das unidades básicas, estando relacionado tanto à falta do profissional quanto à falta de equipamentos e insumos, mas, principalmente, porque na maioria das vezes, existe somente uma 
equipe de saúde bucal para cada duas equipes de saúde da família, dificultando, dessa forma o acesso da população aos atendimentos odontológicos (Lima et al., 2018). O número inferior de equipes de saúde bucal, se comparado ao número de equipes de saúde da família, tem ao longo do tempo, prejudicado a oferta de serviços importantes da atenção básica, que devese pautar, priomordialmente, na prevenção de doenças e promoção da saúde.

A existência de uma grande demanda reprimida em odontologia é decorrente da falta de assistência odontológica durante muitas décadas da qual decorre uma enorme procura por procedimentos curativos, dificultando a reversão do modelo assistencial-tecnicista (Barros et al., 2016).

De acordo com Warmling; Baldisserotto; Rocha (2018), o problema no modelo de práticas baseado quase que exclusivamente no atendimento às queixas dos usuários que buscam as unidades de saúde, é a sua forma organizar excludente, priorizando grupos específicos em detrimento da maioria da população.

No que diz respeito à organização da demanda, observa-se que essa é feita de acordo com a disponibilidade do serviço e dos profissionais existentes das equipes de saúde da família.

Mattos et al., (2014) relata que a forma de organizar o atendimento odontológico ocorre de duas formas: através da demanda organizada e através da demanda espontânea caracterizando o pronto atendimento. Os autores relatam que o agendamento pode ocorrer através dos Agentes Comunitários de Saúde e em outras situações pode ser realizado através do Auxiliar de Saúde Bucal, em um dia previamente marcado na unidade de saúde ou nas escolas.

Em pesquisa realizada com as equipes de saúde bucal de Porto Alegre, foi observado que o ato de acolher as necessidades de saúde bucal está centrado em promover acesso ao atendimento odontológico, de maneira que é realizado mediante a demanda espontânea de urgências, conforme agendamento tradicional por meio da distribuição de fichas individuais, ou ainda por organização de grupos com identificação de necessidades. Ocorre uma valorização da conduta terapêutica fundamentada na definição de número de vagas para o atendimento de procedimentos odontológicos necessários para a conclusão de planos de tratamento (Warmling; Baldisserotto; Rocha, 2018).

O acolhimento está intimamente ligado ao acesso dos usuários ao serviço de saúde. De acordo com Moimaz et al., (2017) a oferta de procedimentos pela equipe e a disponibilidade dos profissionais para dialogar sobre dúvidas, preocupações e problemas de saúde bucal foram os fatores que mais destacaram-se na qualificação dos indicadores do acolhimento no serviço odontológico do Brasil.

No estudo de Mattos et al., (2014) os Cirurgiões-dentistas e as Auxiliares em Saúde Bucal (ASB) relataram uma transformação do acesso dos usuários aos serviços odontológicos, que passaram a ser agendados. Essa mudança no acesso através do agendamento ocorreu concomitantemente ao aumento numérico dos profissionais de saúde e Equipes de Saúde Bucal.

O acolhimento não está restrito apenas à entrada do usuário no sistema de saúde, mas a responsabilização integral de suas necessidades de maneira resolutiva dos seus problemas. A partir desse entendimento ampliado, o acolhimento poderá provocar a reorganização do serviço ao atender positivamente as demandas e anseios em saúde dos usuários (MOIMAZ et al., 2017).

Nessa perspectiva, o acolhimento em saúde bucal, faz-se necessário, como instrumento de gestão e atenção do cuidado, por ser uma ferramenta importante que auxilia não apenas na classificação de risco dos usuários, mas também proporciona resolutividade e integralidade.

Alguns dos estudos dessa revisão trouxeram a visita domiciliar como uma oportunidade de avaliação e atenção da saúde bucal de pacientes, especialmente aqueles com dificuldade de locomoção (Moraes; Klingerman; Cohen, 2015).

A visita domiciliar é um instrumento valoroso utilizado pela Equipe de Saúde da Família que possibilita o estabelecimento de vínculos com os usuários, reconhecendo o cotidiano da comunidade e as relações entre os familiares. Nesta 
ação devem estar envolvidas atividades de promoção da saúde, prevenção de agravos e o acompanhamento familiar, sendo necessário o planejamento e o registo pela equipe de saúde. Vale ressaltar que a assistência domiciliar deve ser prestada a pacientes com dificuldade de mobilidade, enfermos acamados temporariamente ou definitivamente, podendo pertencer a qualquer faixa etária, não havendo uma patologia ou grupo de patologias específico (Ferraz; Leite, 2016).

A necessidade de encaminhamento para outros pontos de atenção ficou evidenciado nos estudos (Cavalcanti et al., 2012; Fagundes et al., 2018; Reis et al., 2019; Rodrigues et al., 2010), em concordância com Souza et al., (2015), que relatam que o paciente ao ser encaminhado para o CEO (Centro de Especialidades Odontológicas) pelo dentista da Atenção Básica possui maior probabilidade de voltar a esse nível de atenção para a continuidade do tratamento, demonstrando que a referência e a contrarreferência através do encaminhamento contribui para a integralidade do cuidado à saúde.

O crescimento das ações em odontologia no âmbito da Atenção Primária à Saúde promoveu um aumento da demanda por consultas e tratamentos especializados e os CEO (Centros de Especialidades Odontológicas) não conseguem absorver completamente os usuários referenciados (Aquilante; Acioli, 2013).

As mudanças do modelo de saúde bucal centradas, prioritariamente, no curativo perpassam por ferramentas de organização e mudanças no processo de trabalho em busca da integralidade do serviço.

O processo de trabalho em saúde bucal, de acordo com Neves et al., (2017) não se limita na assistência odontológica individual, por sua vez, a integralidade só é alcançada através da criação de redes macro e microinstitucionais, em processos dinâmicos, voltados ao fluxo de assistência ao usuário e centrada em seu campo de necessidade.

Os odontólogos da Atenção Primária produzem cuidado à saúde baseados em atuações clinicas fundamentadas no acolhimento, na escuta e no diálogo com os usuários, com a consolidação de aspectos subjetivos, promovendo a pactuação de um plano de tratamento compartilhado e em contínua edificação. Esses profissionais possuem uma percepção do cuidado divergente das práticas assistenciais (Graffi; Toassi, 2018).

O modelo de organização da atenção básica deve ser repensado com ferramentas de telefone e internet para facilitar o agendamento de consultas e favorecer o vínculo entre a unidade e o usuário, buscando aumentar e garantir o acesso e a resolutividade das unidades básicas brasileiras (Lima et al., 2018).

Outra questão que deve valorizada, diz respeito à percepção de profissionais com uma compreensão de cuidado contrária a uma assistência com foco essencialmente dentário a partir de necessidades diagnosticadas exclusivamente pelo profissional. A subjetividade deve expressar-se pelo reconhecimento do usuário como um sujeito de experimentação e interação social, cujas necessidades do corpo vivido vão além das de um corpo físico (Graff; Toass, 2018).

Dessa forma, é importante ressaltar que um conjunto de ações estruturadas para fortalecimento da Atenção Primária à Saúde é fundamental para se obter eficiência. Isso passa por uma rede de atenção à saúde organizada e com fluxos estabelecidos, melhoria contínua do acesso, investimentos em residências (medicina de família e comunidade e multiprofissional) e a definição de uma carteira de serviços que atenda as necessidades da população (Cunha et al., 2019).

\section{Conclusão}

Os estudos dessa revisão evidenciaram que o acesso às ações de saúde bucal está voltado principalmente às queixas dos pacientes, com procedimentos, na maioria das vezes, curativos e com baixa resolutividade.

O acolhimento é realizado de forma incipiente, não considerando o usuário como sujeito do processo. A falta de profissionais de saúde bucal, apontado em alguns estudos, mostrou-se como limitação para atenção de forma integral na atenção primária à saúde. As atividades de educação em saúde e prevenção, quando ocorrem, são realizadas em escolas e direcionadas às crianças e adolescentes. 
Diante dos resultados apresentados, revela-se que o quesito integralidade na atenção básica em odontologia encontrase fragilizado pela dificuldade de acesso, falha na organização da demanda, carência de educação e promoção da saúde, bem como limitação na continuidade de tratamento.

Faz-se necessária uma reformulação dos currículos dos cursos de graduação em odontologia e a promoção de cursos para os profissionais atuantes nas Equipes de Saúde Bucal para que ocorra a mudança no paradigma curativo-mutilador, centrado na doença em detrimento do modelo preventivo, reabilitador e promotor da saúde proposto pelo Sistema Único de Saúde para que a população brasileira possa ser atendida com qualidade, eficiência, efetividade e resolutividade em relação aos seus problemas de saúde bucal, evitando agravos e melhorando a qualidade de vida dos usuários.

Por outro lado, é importante maiores investimentos dos governos e da gestão na saúde bucal, com melhor estruturação das equipes, aquisição de equipamentos e materiais, contratação de profissionais, valorização profissional, organização da rede de serviços e ampliação das equipes de saúde bucal no país, de forma equiparada às equipes de saúde família.

$\mathrm{O}$ conceito de integralidade do cuidado à saúde é abrangente e polissêmico traduzindo-se em aspectos variados da atenção em saúde, como o acolhimento, vínculo-responsabilização e qualidade da atenção, o que certamente dificulta sua caracterização de forma precisa nos estudos, estando permeado por esses atributos que conjuntamente somados devem resultar no cuidado integral preconizado pelo SUS.

Outra limitação do estudo refere-se à pequena quantidade de estudos realizados com os usuários do SUS, comprometendo uma análise da integralidade sob a ótica de quem recebe a atenção em saúde.

\section{Referências}

Aquilante, A. G., Aciole, G. G. (2015). O cuidado em saúde bucal após a Política Nacional de Saúde Bucal - "Brasil Sorridente": um estudo de caso. Revista Ciência \& Saúde Coletiva. 20 (1): 239-248. https://www.redalyc.org/pdf/630/63033062027.pdf.

Brasil. (2000). Ministério da Saúde. Portaria MS n. 1.444 de 28 de dezembro de 2000. Estabelece incentivo financeiro para a reorganização da atenção à saúde bucal prestada nos municípios por meio do Programa de Saúde da Família. Diário Oficial da União. Brasília. http://www1.saude.rs.gov.br/dados/11652497918841\%20Portaria\%20N\%BA\%201444\%20de\%2028\%20dez\%20de\%202000.pdf.

Brasil. (2004). Ministério da Saúde. Secretaria de Atenção à Saúde. Departamento de Atenção Básica Coordenação Nacional de Saúde Bucal. Diretrizes da Política Nacional de Saúde Bucal. Brasília: Ministério da Saúde. http://bvsms.saude.gov.br/bvs/publicacoes/politica_nacional_brasil_sorridente.htm.

Barros, S. G., et al. (2016). Impacto da implantação das equipes de saúde bucal na estratégia saúde da família em dois municípios do estado da Bahia. Revista de Saúde Coletiva. 6(2): 37-42. http://periodicos.uefs.br/index.php/saudecoletiva/article/view/1212.

Casotti, E., et al. (2014). Atenção em Saúde Bucal no Brasil: uma análise a partir da Avaliação Externa do PMAQ-AB. Saúde debate. 38, <http://www.scielo.br/scielo.php?script=sci_arttext\&pid=S0103-11042014000600140\&lng=en\&nrm=iso>.

Cavalcanti, Y. W., et al. (2012). Avaliação de usuários da Atenção Básica sobre a Implantação de Protocolo de Assistência Integral Odontológica. Revista Pesquisa Brasileira em Odontopediatria e Clinica Integrada, 12(3):405-12, https://www.redalyc.org/pdf/637/63770311.pdf.

Cunha, C. R. H., et al. (2020). Carteira de Serviços da Atenção Primária à Saúde: garantia de integralidade nas Equipes de Saúde da Família e Saúde Bucal no Brasil. Revista Ciência \& Saúde Coletiva, 25(4):1313-1326. https://www.scielo.br/scielo.php?script=sci_arttext\&pid=S1413-81232020000401313. Acesso em 26 de março de 2021.

Fagundes, D. M., et al. (2018). Diálogos sobre o processo de trabalho em saúde bucal no Brasil: uma análise com base no PMAQ-AB. Revista Cad. Saúde Pública; 34(9): e00049817https://www.scielo.br/pdf/csp/v34n9/1678-4464-csp-34-09-e00049817.pdf.

Ferraz, G. A., Leite, I. C.G. (2016). Instrumentos de visita domiciliar: abordagem da odontologia na estratégia saúde da família. Revista APS. 19 (2): 302 - 314. https://periodicos.ufjf.br/index.php/aps/article/view/15647.

Graff, V. A., Toassi, R. F. C. (2018). Clínica em saúde bucal como espaço de produção de diálogo, vínculo e subjetividades entre usuários e cirurgiõesdentistas da Atenção Primária à Saúde. Revista de Saúde $28(3)$ Coletiva, 280313. https://www.scielo.br/scielo.php?pid=S010373312018000300608\&script=sci_abstract\&tlng=pt.

Lima, J. G., et al. (2018). Atributos essenciais da Atenção Primária à Saúde: resultados nacionais do PMAQ-AB. Revista Saúde Debate. 42, 52-66, http://www.scielo.br/pdf/sdeb/v42nspe1/0103-1104-sdeb-42-spe01-0052.pdf.

Maciel, J. A. C., et al. (2020). Avaliação de usuários sobre atributos da atenção primária à saúde bucal na Estratégia Saúde da Família. Revista APS. http://www.scielo.br/pdf/sdeb/v42nspe1/0103-1104-sdeb-42-spe01-0052.pdf.

Mattos, G. C. M., et al. (2014). A inclusão da equipe de saúde bucal na Estratégia Saúde da Família: entraves, avanços e desafios. Revista Ciência \& Saúde Coletiva, 19(2): 373-382. https://www.scielo.br/scielo.php?pid=S1413-81232014000200373\&script=sci_abstract\&tlng=pt. 
Melo, L. M. L., et al. (2016). Avaliação da saúde bucal na ótica do gestor municipal. RGO - Revista Gaúcha de Odontologia, 64(4) https://www.scielo.br/j/rgo/a/c9b3cKkJX3gTPzb4CVrSFPz/abstract/?lang=pt.

Mendes, K. D. S., Silveira, R. C. C. P., Galvão, C. M. (2008). Revisão Integrativa: método de pesquisa para a incorporação de evidências na saúde e na enfermagem. Texto ContexEnferm, .17(4), 758 - 764. http://www.scielo.br/scielo.php?pid=s010407072008000400018\&script=sci_abstract\&tlng=pt.

Moher, D., et al. (2009). PRISMA Group. Preferred reporting items for systematicreviews and meta-analyses: the PRISMA statement. BMJ. Jhttps://www.google.com/aclk?sa=1\&ai=DChcSEwiqt6iov43xAhWQhcgKHTsAArIYABAAGgJxdQ\&ae=2\&sig=AOD64_1Qz1TDLqkoXa_MfCvp5PRRkb D_dQ\&q\&adurl\&ved=2ahUKEwjy8o-ov43xAhV6HLkGHVOxBLAQ0Qx6BAgCEAE..

Moimaz, A. S., et al. (2017). Qualificação do acolhimento nos serviços de saúde bucal. Caderno de Saúde Coletiva, 25 [1]: 1-6. http://www.scielo.br/pdf/cadsc/v25n1/1414-462X-cadsc-25-1-1.pdf.

Moraes, L. B., Kligerman, D. C., Cohen, S. C. (2015). Análise do perfil sociodemográfico e do processo de trabalho do cirurgião-dentista inserido no Programa de Saúde da Família em três municípios da região serrana do Estado do Rio de Janeiro. Revista de Saúde Coletiva, 25 [1]: 171-186 http://www.scielo.br/scielo.php?script=sci_arttext\&pid=S0103-73312015000100171.

Munkeviz, M. S. G., Pelicioni, M. C. F. (2010). Saúde bucal na estratégia saúde da família no município de São Paulo: perspectiva do usuário. Revista Brasileira de Crescimento Desenvolvimento Humano. 20(3) 791-801. https://www.revistas.usp.br/jhgd/article/view/19987.

Neves, M., Giordani, J. M.A., Hugo, F. N. (2019). Atenção primária à saúde bucal no Brasil: processo de trabalho das equipes de saúde bucal. Revista Ciência \& Saúde Coletiva, 24 (5): 1809-1820. https://www.scielo.br/scielo.php?script=sci_abstract\&pid=S1413-81232019000501809\&lng=en\&nrm=iso\&tlng=pt.

Reis, C. M. R., et al. (2019). Understanding oral health care team performance in primary care: A mixedmethod study. PLOS ONE I https://doi.org/10.1371/journal.pone.0217738 https://www.arca.fiocruz.br/bitstream/icict/35006/2/Understanding\%20oral\%20health\%20care\%20team.pdf.

Rodrigues, A. A. A. O., et al. (2010). Práticas da equipe de saúde bucal na estratégia saúde da família e a construção (des) construção da integralidade em Feira de Santana - BA. Rev. APS, 13, 476-485, https://periodicos.ufjf.br/index.php/aps/article/view/14676. .

Sampaio R.F., Mancini, M.C. (2007). Estudos de revisão sistemática: um guia para síntese criteriosa da evidência científica. Revista Brasileira de Fisioterapia, São Carlos, 83-89, https://www.scielo.br/j/rbfis/a/79nG9Vk3syHhnSgY7VsB6jG/?lang=pt.

Santos, A. M., Assis, M. M. A. (2006). Da fragmentação à integralidade: construindo e (des)construindo a prática de saúde bucal no Programa de Saúde da Família (PSF) de Alagoinhas, BA. Revista Ciência \& Saúde Coletiva, 11(1):53-61. https://www.scielo.br/scielo.php?pid=S1413$81232006000100012 \&$ script=sci_abstract\&tlng=pt.

Silva, M. A. M., Figueiredo, A. C. M., Gueiros, R. F. (2020). Diferentes olhares sobre os serviços de saúde bucal no SUS. Researchgate, https://www.researchgate.net/publication/338657453_RELATORIO_TECNICO_Titulo_Diferentes_olhares_sobre_os_servicos_de_saude_bucal_no_SUS?enri chId=rgreq-1dd81bef4060eb76cc11d5c58a154f39-XXX\&enrichSource=Y292ZXJQYWdiOzMzODY1NzQ1MztBUzo4NDgzNjk5ODM0MjY1NjRAMTU3 OTI3ODU3NDU 1MA\% 3D\%3 D\&el=1_x_2\&_esc=publicationCoverPdf.

Silva, M. F. F., et al. (2018a). Integralidade na atenção primária à saúde. Revista Família, Ciclos de Vida e Saúde no Contexto Social, vol. 1. Universidade Federal do Triângulo Mineiro, Brasil. http://www.redalyc.org/articulo.oa?id=497955551016.

Silva, S. E., et al. (2018b). Caracterização do modelo de atenção básica à saúde bucal na região nordeste no período de 2015-2017. Arch. Health Invest ; 7(10): 402-407. https://pesquisa.bvsalud.org/portal/resource/pt/biblio-994522.

Solano, M.P., et al. (2017). Revista Ciência Plural. 3(3):81-92. https://periodicos.ufrn.br/rcp/article/view/13303.

Souza, G. C., et al (2015). Referência e contra referência em saúde bucal: regulação do acesso aos centros de especialidades odontológicas. Revista salud pública. 17 (3): 416-428. https://scielosp.org/article/rsap/2015.v17n3/416-428/.

Warmling, C. M., Baldisserotto, J., Rocha, E. T. (2019). Acolhimento \& acesso de necessidades de saúde bucal e o agir profissional na Atenção Primária à Saúde. Revista Interface. Botucatu. 23: e180398. https://doi.org/10.1590/Interface.180398. 